\title{
Formation of Porous Structure with Subspot Size under the Irradiation of Picosecond Laser Pulses
}

\author{
Bin Liu, Wenjun Wang, Gedong Jiang, Xuesong Mei, Kedian Wang, and Jiuhong Wang \\ State Key Laboratory for Manufacturing System Engineering, Xian Jiaotong University, Xian 710054, China \\ Correspondence should be addressed to Wenjun Wang; wenjunwang@mail.xjtu.edu.cn
}

Received 17 July 2013; Revised 6 September 2013; Accepted 8 September 2013

Academic Editor: Guangyu Zhao

Copyright (C) 2013 Bin Liu et al. This is an open access article distributed under the Creative Commons Attribution License, which permits unrestricted use, distribution, and reproduction in any medium, provided the original work is properly cited.

A study was presented in this paper on porous structure with microsize holes significantly smaller than laser spot on the stainless steel 304 target surface induced by a picosecond Nd:van regenerative amplified laser, operating at $1064 \mathrm{~nm}$. The target surface variations were studied in air ambience. The estimated surface damage threshold was $0.15 \mathrm{~J} / \mathrm{cm}^{2}$. The target specific surface changes and phenomena observed supported a complementary study on the formation and growth of the subspot size pit holes on metal surface with dependence of laser pulse number of 50-1000 and fluences of 0.8 and $1.6 \mathrm{~J} / \mathrm{cm}^{2}$. Two kinds of porous structures were presented: periodic holes are formed from Coulomb Explosion during locally spatial modulated ablation, and random holes are formed from the burst of bubbles in overheated liquid during phase explosion. It can be concluded that it is effective to fabricate a large metal surface area of porous structure by laser scanning regime. Generally, it is also difficult for ultrashort laser to fabricate the microporous structures compared with traditional methods. These porous structures potentially have a number of important applications in nanotechnology, industry, nuclear complex, and so forth.

\section{Introduction}

The application of lasers surface structure fabrication to different materials including metal is of great interest. With the appearance of ultrashort laser in 1990s, material/metal surface texturing gained a whole new dimension. From that moment, it became possible to touch three time regimes from fs, ps, to ns [1]. Surface structure fabrications by ultrashort laser have been paid serious attention because it can manufacture structures with sub-spot size and can provide greater flexibility in fixing the position of ablated areas $[2,3]$. They have widely potential applications in the fields of physics, chemistry, and materials, such as enhancing light absorption [4], improving catalytic action [5], and strengthening tribological and hydrophilic properties [6].

In the recent years, the fabrication of the micro- and nanosized periodical structures on the surface of materials by laser radiation with intensity exceeding the melt threshold has been intensively studied [7-9]. Periodic patterns of the ripples are often referred to as one of the most common laser-induced periodic surface structures (LIPSS). Some pit holes with diameters significantly smaller than laser spot are often observed during LIPSS fabrication on material surface
$[10,11]$. So far, many studies have been carried out on LIPSS formed by laser pulses [7-9]. However, a detailed study on the origin and development of these pit holes is rare, but some underlying physical effects have been preliminarily investigated by pump-probe microscopy on $\mathrm{Ta}_{2} \mathrm{O}_{5} / \mathrm{Pt}$ layer system on glass substrate [12]. Fan and Zhong found that specific porous coral-like structures on $\mathrm{Cu}$ by picosecond can enhance broadband absorption [13], and Zhao and Zhang found that the porous structure on $\mathrm{MoO}_{3}$ films can be used for supercapacitors [14].

In this work, a complementary study was presented on the influence of the laser pulse number and fluence on the formation and growth of these subspot size pit holes on metal surface. Nano/micrometer-sized holes were found to be formed under specific irradiation conditions. A comparison of these experimental results based on the currently accepted mechanism revealed the evolution of the holes.

\section{Experimental}

The laser system (High-Q IC-1500) based on a regenerative Nd:van amplifier using chirped pulse amplification technique 


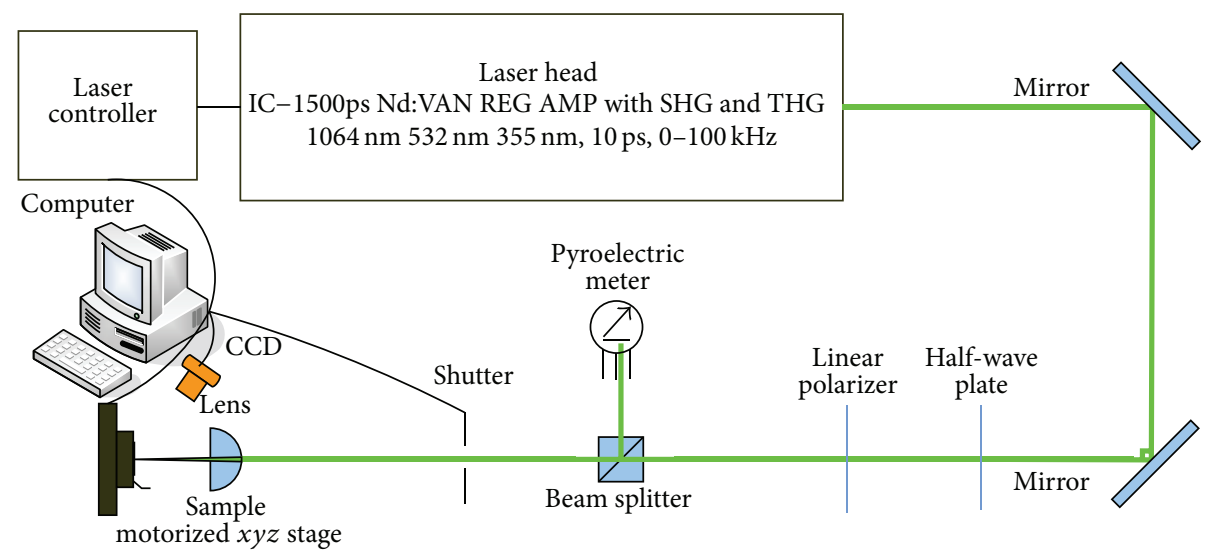

FIgURE 1: Experimental device.

provides high-intensity picosecond laser pulses for the experiment. The pulse duration of the output beam from amplifier is $10 \mathrm{ps}$ with nominal wavelength at $1064 \mathrm{~nm}$. The laser system delivers $\sim 0.3 \mathrm{~mJ}$ per pulse at $1 \mathrm{kHz}$ repetition rate and $\lambda=$ $1064 \mathrm{~nm}, 10 \mathrm{ps}$ pulse length, and observed bandwidth $\Delta \lambda$ $\sim 5.5 \mathrm{~nm}$. The power density distribution of the laser beam is Gaussian with a measured 3D Gaussian fit parameter of $\sim 0.87$. The IR ( $1064 \mathrm{~nm})$ radiation can be converted into green (532 nm) by a Second Harmonic Generator (SHG) unit. The experimental setup is depicted in Figure 1. The laser power is adjusted by using a combination of a half-wave plate and a linear polarizer. A pyroelectric detector is used to inspect the laser power in real time with a beam splitter. The number of pulses is programmable using an electromechanical shutter control. The laser beam are focused and normally irradiated onto the surface of sample using $f$-theta lens $\left(f^{\prime}=150 \mathrm{~mm}\right)$. A motorized $x y z$ stage controlled by computer is used to get a precise positioning of the samples.

The experiment was performed on the stainless steel 304 which is widely used in industry. Firstly, the surface of the steel sample was mechanically polished with sandpaper with a grit size of 2500 grooves $/ \mathrm{mm}$ and subsequently fine polished with the Alumina powder with an average particle size of $\sim 500 \mathrm{~nm}$. After the polishing process, the sample was cleaned in an ultrasonic bath firstly by acetone to remove oily contaminants for 10 minutes and subsequently by alcohol for 10 minutes. Finally, the sample was rinsed with distilled water and dried by nitrogen.

In experiments, all the laser ablation experiments were performed in air. The samples were irradiated by a series of laser pulses with the different fluence and pulse number at wavelength $1064 \mathrm{~nm}$ and $1 \mathrm{kHz}$ repetition rate. After laser irradiation, the ablated surfaces of the samples were inspected with a Scanning Electron Microscope (SEM: Hitachi SU8010) and Energy Dispersive X-ray (EDX) which is the component of SEM. For a detailed 3D characterization of multipulse laser ablated craters, Laser Confocal Scanning Microscopy (LCSM: Olympus OLS-400) imaging was used.

\section{Results and Discussions}

The surface of the stainless steel 304 had a typical silvergrey metallic color before irradiation. The investigation of the effect of picosecond laser on target morphological changes was started with a series of the spot irradiated by different pulse numbers $N$ and fluences $F$. It was interesting to find that some sub-spot holes are formed at the bottom of some ablated crater under the certain situations.

3.1. Generation of Holes Depended on Laser Fluence. Figure 2 shows SEM images of craters ablated by picosecond laser with fluences $F=0.8$ and $1.6 \mathrm{~J} / \mathrm{cm}^{2}$ and the pulse number $N=500$. The specific surface morphology of the ablated craters can be observed in SEM images.

Figure 3 shows LCSM images of ablated craters for laser fluences of 0.8 and $1.6 \mathrm{~J} / \mathrm{cm}^{2}$ with 500 pulses. 3D feature is shown in the LCSM images with typical cross-sectional graphs taken along the crater centre at the right-hand side of each image.

For the fluence of $0.8 \mathrm{~J} / \mathrm{cm}^{2}$, microholes on the irradiated surface are shown in Figures 2(a) and 3(a). These pit holes varied in dimension with the dependence of the Gaussian laser energy distribution field. Holes with the average diameters of $\sim 1.5 \mu \mathrm{m}$ are observed in the center of ablated crater (lefthand inset SEM images in Figure 2(a)); while near the edge of ablated crater, pit holes with the average diameter of $\sim 0.9 \mu \mathrm{m}$ also appear among the ripples (right-hand inset SEM images in Figure 2(a)). All holes seem to be regularly lying along lines which are directed almost perpendicular to the ripples, and the average distance between two adjacent holes $\sim 3.6 \mu \mathrm{m}$ led to hole-lying lines with period $\sim 3.6 \mu \mathrm{m}$. These periodic holes were also observed in previous report while in-depth analysis was absent [11]. At the outer edge of ablated crater, ripples just remain because of the lower fluence. The depth of ablated crater reaches $\sim 7 \mu \mathrm{m}$, but the depth of pit holes in the center of ablated crater bottom is very small.

When the fluence increased to $1.6 \mathrm{~J} / \mathrm{cm}^{2}$, morphology of the microholes had a significant change as shown in Figures 2(b) and 3(b); the maximum size of holes in center of ablated crater reaches $\sim 5 \mu \mathrm{m}$ (left-hand inset SEM images in Figure 2(b)). Holes are nonperiodic in the center of ablated crater, which can be called as random holes. The dimension of random holes decreases to $\sim 2 \mu \mathrm{m}$ near the edge of ablated crater, with dependence of the Gaussian laser energy distribution field. A few periodic holes $\sim 1 \mu \mathrm{m}$ are still observed near the edge of ablated crater (right-hand inset SEM images in 


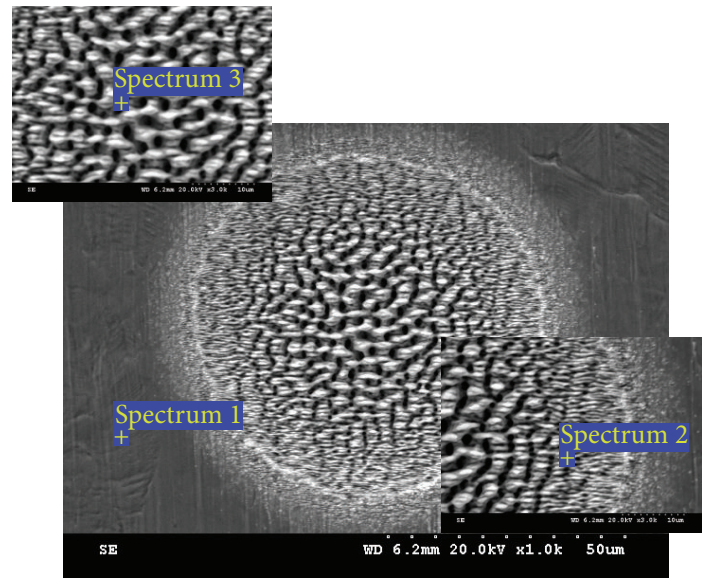

(a)

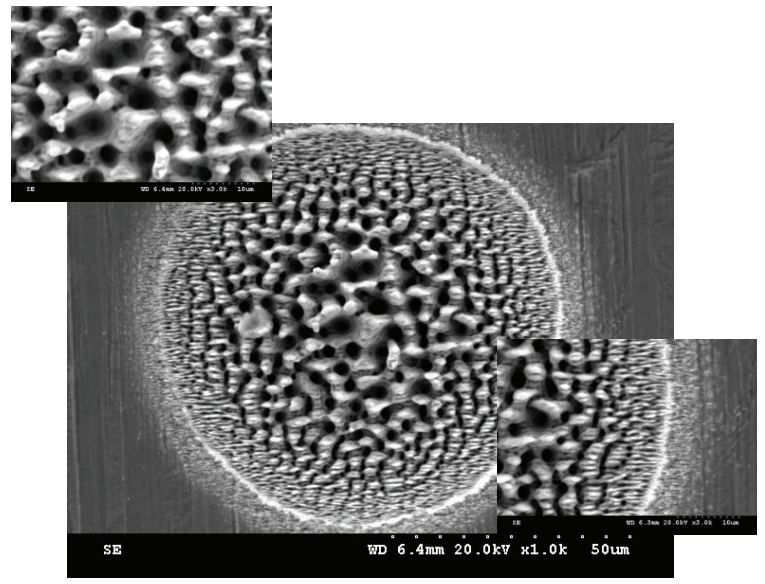

(b)

FIGURE 2: SEM images of 500 pulse ablated craters using fluences of $0.8 \mathrm{~J} / \mathrm{cm}^{2}$ (a) and $1.6 \mathrm{~J} / \mathrm{cm}^{2}$ (b), respectively. The location where pointed spectral analysis was conducted is marked.
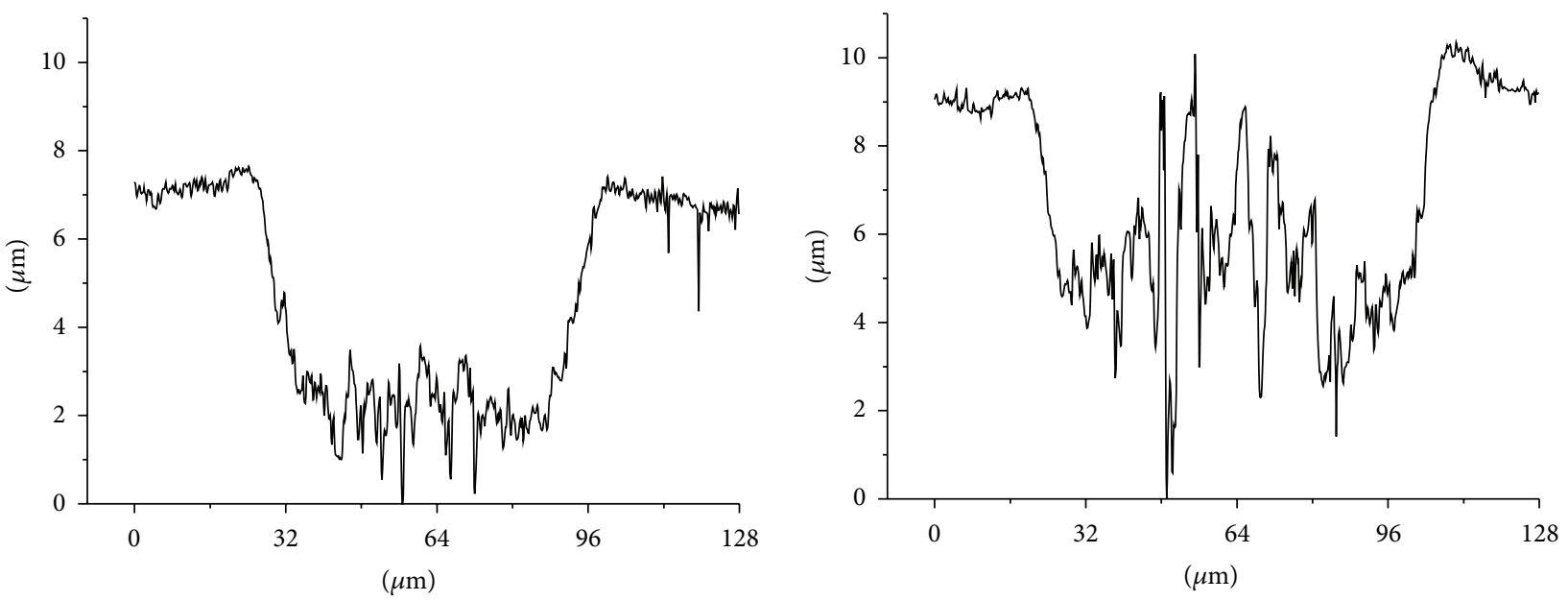

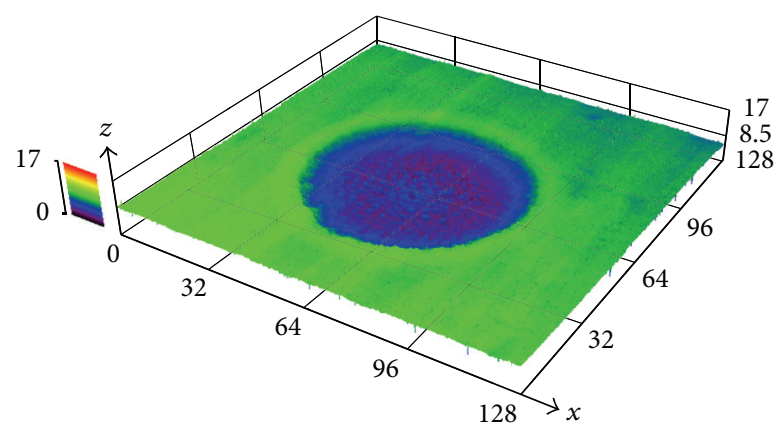

(a)

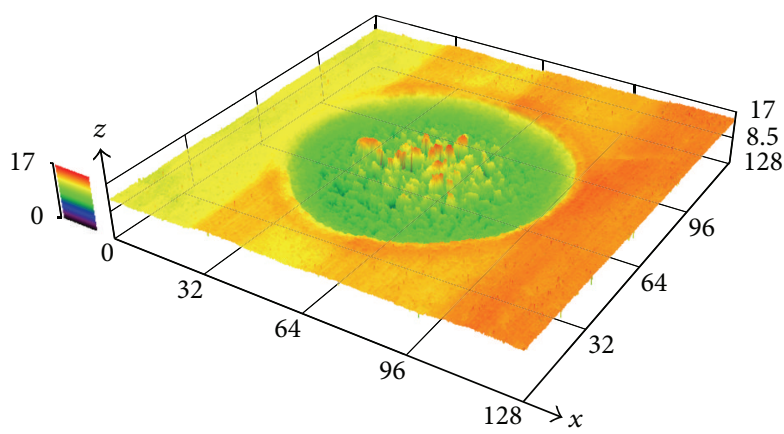

(b)

FIGURE 3: LCSM images of 500 pulse ablated craters using fluences of $0.8 \mathrm{~J} / \mathrm{cm}^{2}$ (a) and $1.6 \mathrm{~J} / \mathrm{cm}^{2}$ (b), respectively.

Figure 2(b)). Compared with Figure 3(a), the average depth of ablated crater seems to be decreasing obviously; however, the depth of microholes on the crater bottom reaches $\sim 9 \mu \mathrm{m}$. A lot of microholes with large depth and diameter make the quality of the crater bottom worse in roughness, as shown in Figure 3(b). It is also inferred that formation of holes in center of ablated crater in Figure 3(b) may be explained by a mechanism that the rapid cooling and resolidification after overheated liquid explosion which differs from the moderate characterization in Figure 3(a).

Irradiation on the target sample was performed in air atmosphere; therefore, the oxygen content in the irradiated 
region is expected to increase. For monitoring of the target constituents, including oxygen, before and after the irradiation of laser pulses, EDX method was used; and the elemental analysis was carried out along the ablated crater at 3 points in Figure 2(a). The results obtained are given in Table 1. Generally, irradiation of the sample laser resulted in the increase of oxygen content and the decrease of carbon content from the periphery towards the center (Table 1, Figure 2(a)). This result implies a relatively efficient removal of carbon from the steel surface.

As shown in Figures 2 and 3, morphologies of the different pit holes in different laser irradiated regions indicate that the hole-formation significantly depends on laser fluence. So it is necessary to understand the effect of laser fluence.

Damage threshold is defined as the minimum laser energy/fluence necessary for creating detectable damage on the material surface. Based on the micrographs of craters ablated by different numbers of pulses (1-500) and fluences $\left(0.2-1.7 \mathrm{~J} / \mathrm{cm}^{2}\right)$, the damage thresholds of the target were determined for different numbers of pulses, as shown in Figure 4. These damage thresholds and focused beam radius can be evaluated using the method given in references $[10,15$, 16]. The focused beam radius $\omega_{0}$ was calculated to be $\sim 45 \mu \mathrm{m}$.

The region of holes did not obviously increase with the increase of the number of pulses. The surface morphology ablated by 500 pulses was used to predict the formation of pit holes dependence of a Gaussian spatial beam fluence profile, which is schematically presented in Figure 5. The fluence of each ablate crater has a Gaussian profile, shown in Figure $5(\mathrm{a}) . F_{\text {th } 2}$ is the threshold for random pit holes and $F_{\text {th1 }}$ is the threshold for periodic pit holes. $F_{\text {th1 }}$ or $F_{\text {th2 }}$ can be calculated by the following equations:

$$
\begin{gathered}
F(r)=F_{0}^{\mathrm{pk}} \exp \left(-\frac{2 r^{2}}{\omega_{0}^{2}}\right) \\
F_{0}^{\mathrm{pk}}=\frac{2 E_{p}}{\pi \omega_{0}^{2}},
\end{gathered}
$$

where $F_{0}^{\mathrm{pk}}$ is the peak laser fluence (the fluence at the center for $r=0), E_{p}$ is laser pulse energy. Based on the surface morphologies in Figures 2(a) and 2(b), $F_{\text {th1 }}$ and $F_{\text {th2 }}$ are $\sim 0.76 \mathrm{~J} / \mathrm{cm}^{2}$ and $\sim 1.2 \mathrm{~J} / \mathrm{cm}^{2}$, respectively.

During laser interaction with metals, when the laser fluence is significantly higher than multipulse damage threshold, the thermal diffusivity cannot be neglected $[17,18]$, and a series of thermal effects such as melting, vaporization of molten material, and dissociation, can be generated on the target [19]. Here a calculation for thermal effects based on two temperatures in ablation process is presented [1]. Considering the following set of parameters for the stainless steel 304 (density $\rho=7.93 \mathrm{~g} / \mathrm{cm}^{3}$, heat capacity $C_{P}=605 \mathrm{~J} \mathrm{~kg}^{-1} \mathrm{~K}^{-1}$ calculated at an average temperature of $T=1005 \mathrm{~K}$ between room temperature $T_{0}=298 \mathrm{~K}$ and the melting temperature $T_{m}=1713 \mathrm{~K}$ [20], and the thickness $d_{\mathrm{abs}}$ equaled to the laser optical energy penetration depth of $\alpha^{-1}=20 \mathrm{~nm}$ [21]), the relationship between the temperature and the pulse fluence can be obtained using the equation of $\Delta T=F /\left(\rho C_{P} d_{\mathrm{abs}}\right)[10]$. During laser acting on the thick material surface layer, only

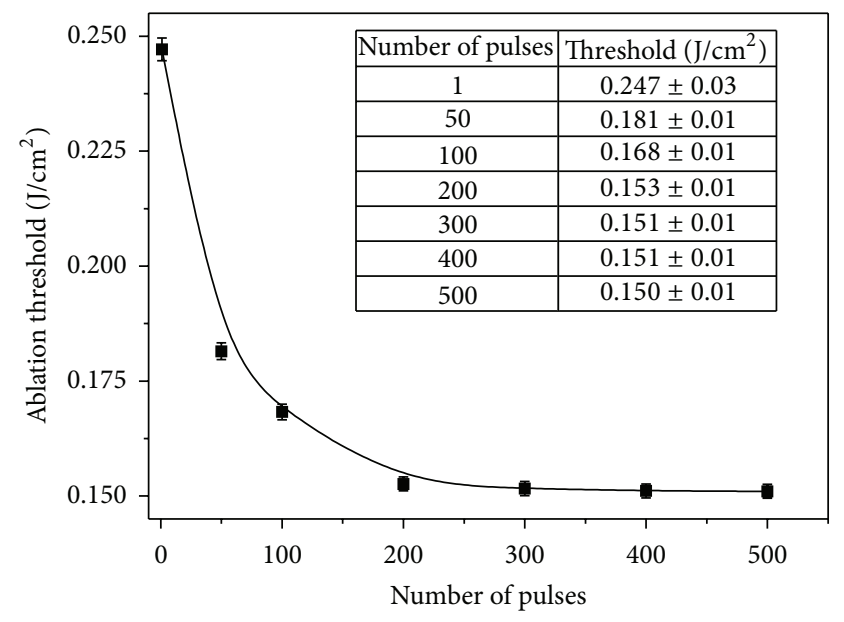

FIgURE 4: Ablation thresholds versus number of pulses.

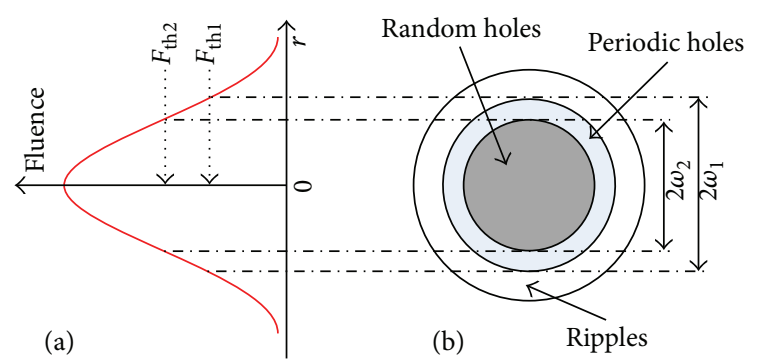

FIGURE 5: Illustration of laser ablation: (a) the profile of Gaussian beam intensity in $r$-axis and (b) the exposure area of ps-laser.

about $10 \%$ of the incidence energy fluence is absorbed [22], and the rest is used for the expanding plasma to move into the ambient air with a small part lost in radiation $[23,24]$. Thus, it is easily calculated that the pulse fluence of $0.147 \mathrm{~J} / \mathrm{cm}^{2} \mathrm{can}$ cause surface melting and the fluence of $0.283 \mathrm{~J} / \mathrm{cm}^{2}$ can cause surface vaporizing. The theoretically calculated threshold $0.147 \mathrm{~J} / \mathrm{cm}^{2}$ agrees with the experimentally calculated threshold $0.150 \mathrm{~J} / \mathrm{cm}^{2}$ well. According to the above analysis, the laser fluences 0.80 and $1.6 \mathrm{~J} / \mathrm{cm}^{2}$ used in Figures 2 and 3 are enough to vaporize the material in skin surface and conduct heat into the bulk of material. Thermal diffusivity indeed occurs.

As the fluence increases from 0.8 to $1.6 \mathrm{~J} / \mathrm{cm}^{2}$, the depth and dimension of pit holes increase. However, the formation of pit holes can be obviously divided into two phases based on the characteristics of pit holes and adopted laser fluences. In phase I, for low fluences $\left(0.76<F<1.2 \mathrm{~J} / \mathrm{cm}^{2}\right)$, more laser energy irradiation makes a growth of periodic pit holes from $\sim 0.9$ to $\sim 1.5 \mu \mathrm{m}$. The formation of these periodic holes may be resulted from Coulomb Explosion [5] during locally spatial modulated ablation. Compared with ripples, the holes have a superiority in absorbing laser energy, which promotes an increasing size of pit holes for prompted dissolution of bubbles of materials after irradiation. In phase II, for high fluences $\left(F>1.29 \mathrm{~J} / \mathrm{cm}^{2}\right)$, the random pit holes present a characteristic of overheated liquid explosion, which differs from phase I. So the creation of holes in phase II may be explained by the phase explosion $[25,26]$. 


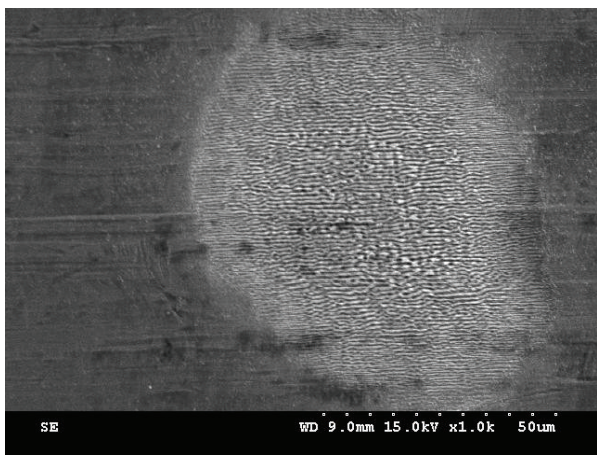

(a)

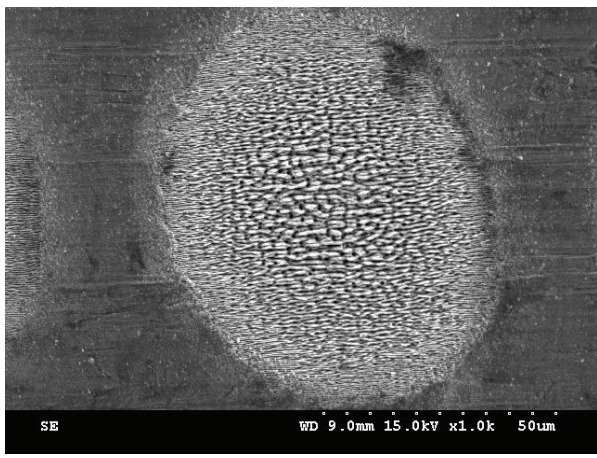

(c)

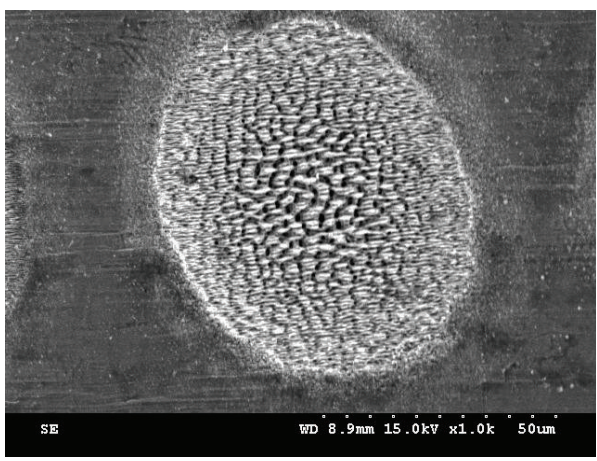

(e)

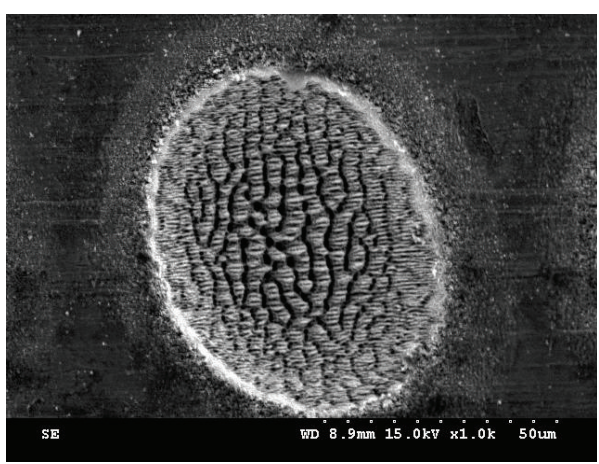

(g)

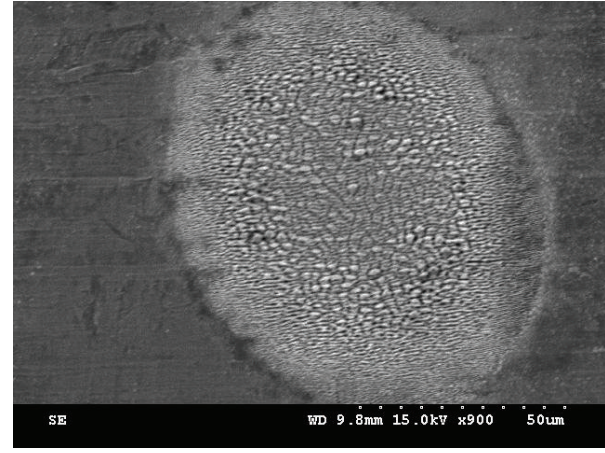

(b)

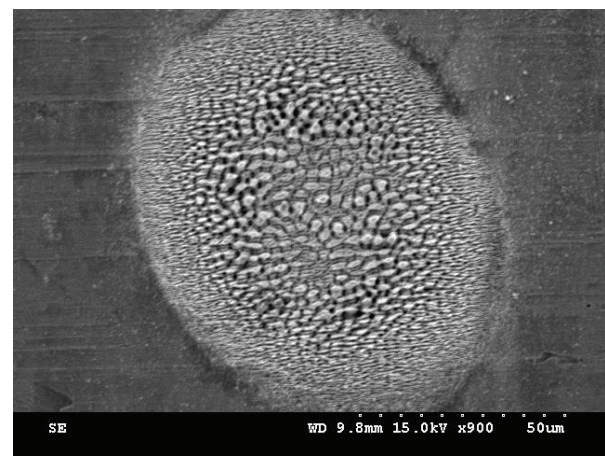

(d)

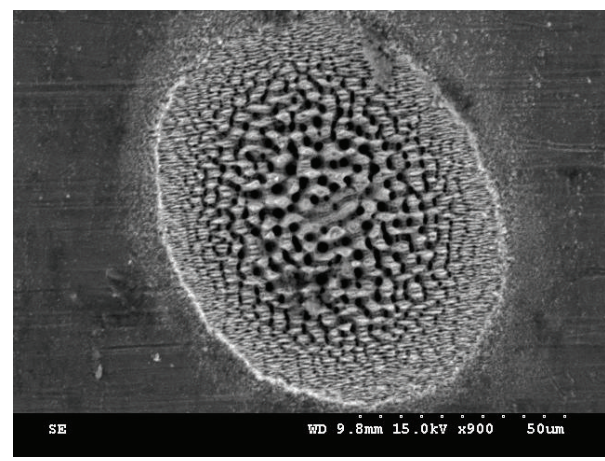

(f)

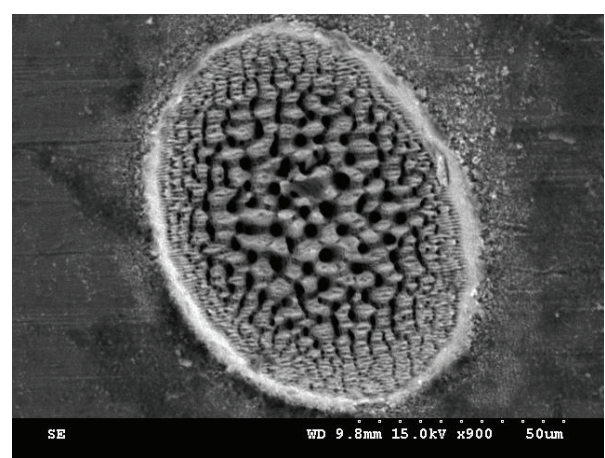

(h)

FIGURE 6: SEM images of an array of craters ablated on the surface of stainless steel 304 with fluence $0.8 \mathrm{~J} / \mathrm{cm}^{2}$ and pulse number of 100 (a), 200 (c), 500 (e), and 1000 (g) and fluence $1.6 \mathrm{~J} / \mathrm{cm}^{2}$ and pulse number of 100 (b), 200 (d), 500 (f), and 1000 (h), respectively. 
TABLE 1: EDX elemental analysis of the stainless steel 304 surface. Measuring locations are given in Figure 2(a).

\begin{tabular}{lcccccccc}
\hline Spectrum (wt.\%) & $\mathrm{C}$ & $\mathrm{O}$ & $\mathrm{Si}$ & $\mathrm{Cr}$ & $\mathrm{Mn}$ & $\mathrm{Fe}$ & $\mathrm{Ni}$ & $\mathrm{Total}$ \\
\hline Spectrum 1 & 8.28 & 2.77 & 0.51 & 16.72 & 1.11 & 62.81 & 7.80 & \\
Spectrum 2 & 5.37 & 5.25 & 0.40 & 17.24 & 1.88 & 62.90 & 6.96 \\
Spectrum 3 & 2.26 & 7.97 & 0.48 & 18.07 & 2.33 & 62.70 & 6.18 \\
\hline
\end{tabular}

(SEM SU-8010, voltage: $15.0 \mathrm{Kv}$, magnification: $10.0 \mathrm{Kx}$, count rate: $1434 \mathrm{cts})$.
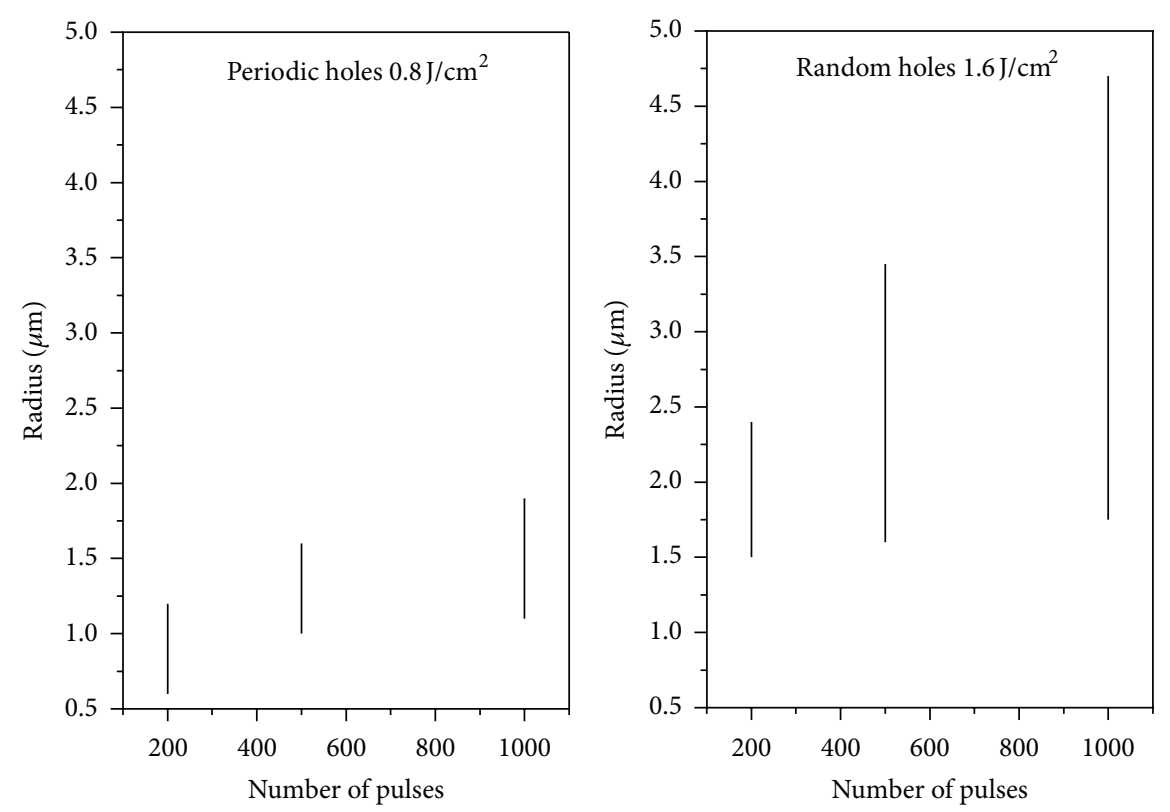

FIGURE 7: Radius of periodic/random holes versus the number of pulses.

3.2. Surface Morphology of Ablated Craters Evolution with Number of Pulses. The section above has discussed two kinds of pit holes, the periodic and random ones, and their causes under the action of different fluences. The following content mainly discusses the role of the pulse number during the formation of pit holes. Figure 6 shows an analysis of the surface morphology of ablated craters irradiated by multiple $(N=50-1000)$ laser pulses with two fluences $(0.8$ and $\left.1.6 \mathrm{~J} / \mathrm{cm}^{2}\right)$. It is found that two kinds of pit hole formation both depend on the number of pulses. As the number of pulses increases from 50 to 500, a transition of main surface structures occurs from ripples (Figures 6(a) and 6(b)) to pit holes (Figures 6(e) and 6(f)). However, due to different mechanisms in two kinds of pit hole formation (discussed in Section 3.1), for $F=0.826 \mathrm{~J} / \mathrm{cm}^{2}$ and $N=$ 1000 , the size of periodic holes increases, and eventually they join together and form channels (Figure 6(g)). These channels also can be deemed a type of periodic surface structure. For $F=1.650 \mathrm{~J} / \mathrm{cm}^{2}$ and $N=200-500$, from Figures 6(d) and 6(f), holes start to form between the center and edge of the ablated crater, whose resolidification is obviously presented in the center; laser with the highest intensity in the center of the beam spot can be mainly used for material removal in the effective penetration depth, which causes less mix of gas-liquid and burst of bubbles in the molten bottom of ablated crater. When $N$ reaches 1000 , the size of random holes also increases and the surface of ablated crater presents porous with resolidification. Images in Figure 6 show that the depth of ablated crater increases obviously but the distribution area of pit holes does not obviously increase with the increasing number of the pulses. Figure 7 shows that the diameter of periodic/random holes increases with the increasing number of the pulses from 200 to 1000 . It is interesting that both of the periodic pit holes and the random pit holes begin to appear at 200 pulses; likewise the ablation threshold tends asymptotically to a constant value at 200 pulses, as shown in Figure 4. It is inferred that the first 200 pulses results in a stable ablated material status and then subsequent pulses have excess energy to reinforce the formation of pit holes.

During laser interaction with metals, the laser energy is absorbed primarily by free electrons [18]. The absorbed radiation energy in the skin layer involves thermalization of electrons. Part of the electron energy is transferred into the bulk in the form of heat, while the later part is transferred to the lattice subsystem. In the first case, the electron diffusion process is essential. Finally, if the initial laser fluence is sufficient, surface damage/ablation will take place. In our experiments, for laser pulses with fluence higher than $0.283 \mathrm{~J} / \mathrm{cm}^{2}$, a single pulse could generate the afterheat, which made a rise of temperature in skin layer of ablated crater. However, the afterheat from a single pulse was not enough for the burst of bubbles in molten material. Seen in Figure 5, the most dramatic decrease of the ablation threshold presents the most dramatic decrease during the first 200 laser pulses: the single 


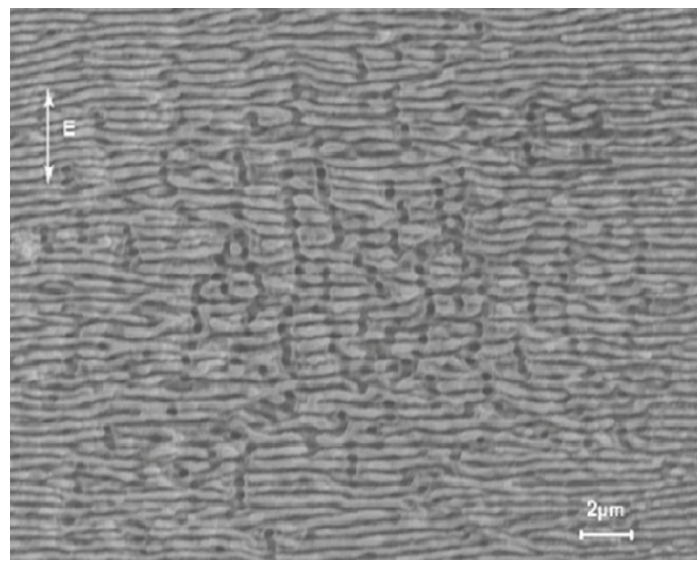

(a)

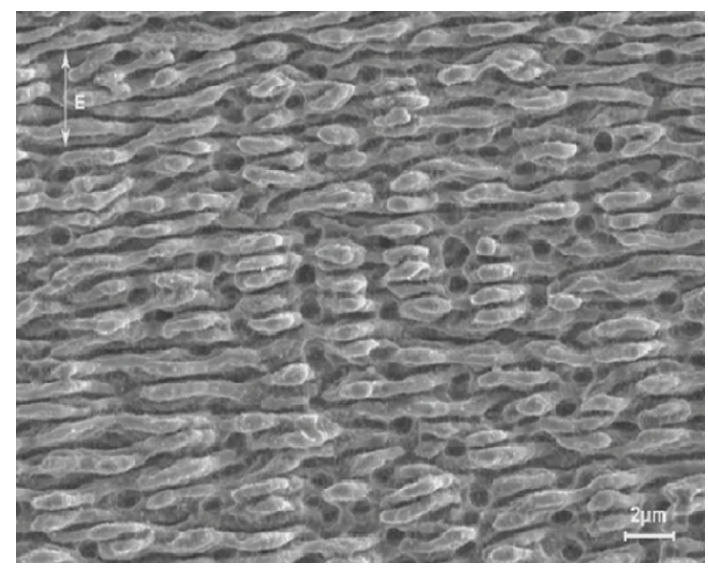

(b)

FIGURE 8: SEM images of surface irradiated by laser pulses of $532 \mathrm{~nm}$ (a) and $1064 \mathrm{~nm}$ (b), with $0.8 \mathrm{~J} / \mathrm{cm}^{2}$ and 250 pluses.

pulse ablation threshold of $0.247 \mathrm{~J} / \mathrm{cm}^{2}$ is reduced to $0.151 \mathrm{~J} /$ $\mathrm{cm}^{2}$, which is an almost $38 \%$ decrease, when 200 laser pulses are applied. As the number of pulses exceeds 200, the threshold fluence tends asymptotically to a constant value of $\sim 0.150 \mathrm{~J} / \mathrm{cm}^{2}$ in this case. After first 200 pulses, the subsequent pulses do not have to use most energy to ablate surface material so that afterheat accumulated promotes the generation of pit holes. So 200 pulses irradiated are necessary for the generation of pit holes. From the different cases among Figures 6(c)-6(f), the optimal number of pulses to form holes for the corresponding fluence needs further study.

3.3. Porous Structures of Ablated Craters Affected by Other Experimental Conditions. The morphological changes induced by picosecond laser on the stainless steel 304 target primarily have shown their dependence on the laser fluence and the number of accumulated pulses, and they are also affected by other factors, such as laser beam characteristics (wavelength, pulse length) and laser irradiation environment (in air atmosphere or in vacuum).

Metals are surface absorbers, with low ionization potential, high electron number density, and higher absorption coefficient for shorter wavelength laser [27]. It means that the morphology of subspot size holes will change with the varying laser wavelength. In order to confirm this inference, the sample surface characteristics are compared after the irradiation of $532 \mathrm{~nm}$ and $1064 \mathrm{~nm}$ wavelength laser, as shown in Figure 8. The experimental result shows the close dependence of the spacing of these periodic holes and the wavelength of incident laser pulses: subspot size holes produced by $532 \mathrm{~nm}$ laser pulses have a period $\sim 1.8 \mu \mathrm{m}$, which is about half of the ones produced by $1064 \mathrm{~nm}$ laser pulses.

In pulsed laser ablation, depending on the respective pulse length range, different beam-matter interaction mechanisms become dominant [1]. For laser pulses in the microand nanosecond range, the ablation process is dominated by heat conduction, melting, evaporation, and plasma formation [19]. Ultrashort pico- and femtosecond laser pulses (where thermal diffusion into the material is small) were used, the generated vapor becomes rapidly ionized [19], and due to the short interaction time, the material cannot evaporate continuously but can be transferred into a state of overheated liquid for the phase explosion [25, 26]. From above, long pulse length may lead to bubbing in molten liquid for mild random holes.

Irradiation of the target sample in this paper was performed in air atmosphere; if in vacuum, the oxidation reaction on the surface can be neglected with absence of the oxide [28]. Ultrafast laser ablation in vacuum leads to the bubbles of materials directly produced from the irradiated material through phase explosion and their flying away from the target surface ahead of the plume with a characteristic velocity of $\sim 10^{4} \mathrm{~m} / \mathrm{s}$ [29]. Therefore, it is inferred that subspot size holes with larger size will be obtained.

3.4. Preparation of Large-Area Porous Structures. In order to explore the effect of porous structures as a kind of functional modified surface structure, the preparation of large-area porous structure is presented. According to the processing parameters discussed above, we choose $F=1.1 \mathrm{~J} / \mathrm{cm}^{2}$ and $V=$ $0.174 \mathrm{~mm} / \mathrm{s}$ (the effective number of pulses in a fixed position during laser scan $N_{f}=500$ ) for a large scanned area of porous structure, as shown in Figure 8. Pit holes are formed on the surface of the scanned area, which have the average diameter of $\sim 5 \mu \mathrm{m}$ (as shown in inset SEM image of Figure 9). And the processing technology for laser scan forming porous structure in large area remains need to be further optimized.

After preparing large-area porous structures, the surface is modified for the hydrophobic surface by fluoroalkyl silane. It is seen that the surface has a contact angle of $151.3^{\circ}$, as shown in Figure 10. Therefore, the porous structure surface has wettability. In addition, porous structure may have many other important applications such as lighting absorption and abrasion resistance, which will be the focus of the future research.

\section{Conclusion}

A study was presented about local morphological changes on the stainless steel 304 target surface induced by a picosecond Nd:van regenerative amplified laser, operating at $1064 \mathrm{~nm}$. 


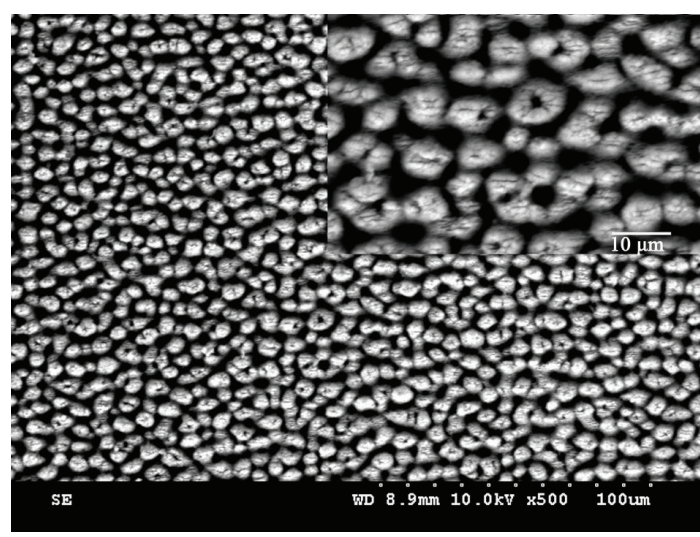

FIGURE 9: SEM image of large-area porous structure fabricated on the surface of stainless steel 304 .
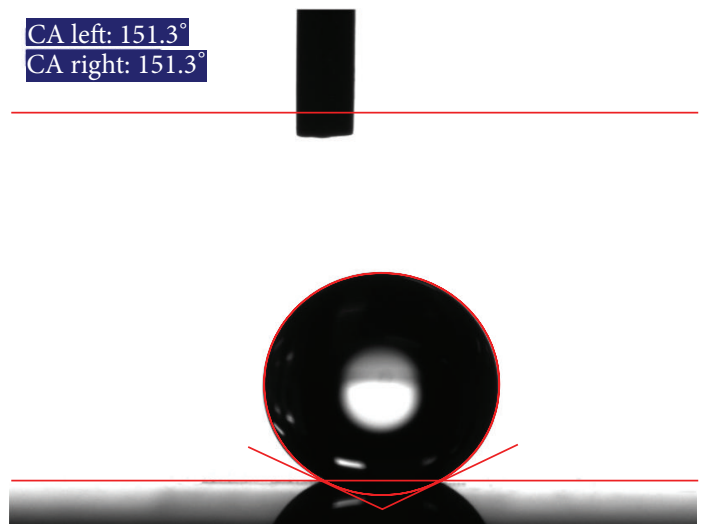

FIGURE 10: Image of contact angle measurements of a water drop on the large-area porous structure surface of stainless steel 304 .

It was shown that laser pulses in the fluences of $\sim 0.8$ and $1.6 \mathrm{~J} / \mathrm{cm}^{2}$ and the numbers of pulses $N=100-1000$, in air, induced specific morphological changes at the stainless steel 304 target. High energy treatment $\left(1.65 \mathrm{~J} / \mathrm{cm}^{2}, 500\right.$ pulses $)$ resulted in the formation of sub-spot hole with the size of $\sim 5-3 \mu \mathrm{m}$, while the reduced energy $\left(0.824 \mathrm{~J} / \mathrm{cm}^{2}, 500\right.$ pulses $)$ led to the creation of sub-spot periodic holes with the size of $\sim 1.5-1 \mu \mathrm{m}$. The experimental results prove that the formation of sub-spot holes on the target depends not only on the number of pulses but also on laser fluence. Although 200 pulse irradiation can cause an initial to form these sub-spot holes, the formation of holes can be obviously divided into 2 phases based on characteristics of holes and energy conditions for their formation. For fluences $\left(0.76<F<1.2 \mathrm{~J} / \mathrm{cm}^{2}\right)$, more irradiating laser energy makes a growth of periodic structures from pits $\sim 300 \mathrm{~nm}$ to holes $\sim 1.5 \mu \mathrm{m}$, finally to periodic groove; for fluences $\left(F>1.2 \mathrm{~J} / \mathrm{cm}^{2}\right)$, the holes present a characteristic of overheated liquid explosion without period and have a larger size.

Generally, it can be deduced that the sub-spot holes with a homogeneous distribution across the whole modified area can have a number of important applications in nanotechnology, industry, nuclear complex, and so forth. Thus, preparation of sub-spot porous structure homogeneously distributed across the whole modified area in large area will be the main direction of our future study.

\section{Acknowledgments}

This work was supported by National Natural Science Foundation of China (Grant no. 91123024), Program for Changjiang Scholars and Innovative Research Team in University (Grant no. IRT1172), and the Fundamental Research Funds for the Central Universities (Grant no. xjj2012113).

\section{References}

[1] B. N. Chichkov, C. Momma, S. Nolte, F. Von Alvensleben, and A. Tünnermann, "Femtosecond, picosecond and nanosecond laser ablation of solids," Applied Physics A, vol. 63, no. 2, pp. 109115, 1997.

[2] W. Wang, X. Mei, and G. Jiang, "Control of microstructure shape and morphology in femtosecond laser ablation of imprint rollers," International Journal of Advanced Manufacturing Technology, vol. 41, no. 5-6, pp. 504-512, 2009.

[3] Y. Ding, J. Shao, X. Li, H. Tian, L. Miao, and H. Liu, "Controllable formation of nanogaps in thin metallic film by rear side irradiation with ultrashort pulsed laser," Physica E, vol. 44, no. 2, pp. 430-434, 2011.

[4] K. Paivasaari, J. J. J. Kaakkunen, M. Kuittinen, and T. Jaaskelainen, "Enhanced optical absorptance of metals using interferometric femtosecond ablation," Optics Express, vol. 15, no. 21, pp. 13838-13843, 2007.

[5] W.-Q. Han, L. Wu, R. F. Klie, and Y. Zhu, "Enhanced optical absorption induced by dense nanocavities inside titania nanorods," Advanced Materials, vol. 19, no. 18, pp. 2525-2529, 2007.

[6] Y. B. Gerbig, S. I.-U. Ahmed, D. G. Chetwynd, and H. Haefke, "Topography-related effects on the lubrication of nanostructured hard surfaces," Tribology International, vol. 39, no. 9, pp. 945-952, 2006.

[7] J. Bonse, A. Rosenfeld, and J. Krüger, "Implications of transient changes of optical and surface properties of solids during femtosecond laser pulse irradiation to the formation of laser-induced periodic surface structures," Applied Surface Science, vol. 257, no. 12, pp. 5420-5423, 2011.

[8] A. Borowiec and H. K. Haugen, "Subwavelength ripple formation on the surfaces of compound semiconductors irradiated with femtosecond laser pulses," Applied Physics Letters, vol. 82, no. 25, pp. 4462-4464, 2003.

[9] G. A. Martsinovskii and G. D. Shandybina, "Ultrashort excitations of surface polaritons and waveguide modes in semiconductors," Optics and Spectroscopy, vol. 105, pp. 67-72, 2008.

[10] N. G. Semaltianos, W. Perrie, P. French et al., "Femtosecond laser ablation characteristics of nickel-based superalloy C263," Applied Physics A, vol. 94, no. 4, pp. 999-1009, 2009.

[11] M. Guillermin, F. Garrelie, N. Sanner, E. Audouard, and H. Soder, "Single- and multi-pulse formation of surface structures under static femtosecond irradiation," Applied Surface Science, vol. 253, no. 19, pp. 8075-8079, 2007.

[12] S. Rapp and J. Rosenberger, "Pump-probe microscopy investigations on fs-laser ablation of thin $\mathrm{Ta}_{2} \mathrm{O}_{5} / \mathrm{Pt}$ layer systems," Journal of Applied Physics, vol. 112, Article ID 013110, 2012.

[13] P. Fan and M. Zhong, "Rapid fabrication of surface micro/nano structures with enhanced broadband absorption on $\mathrm{Cu}$ by picosecond laser," Optics Express, vol. 21, pp. 11628-11637, 2013. 
[14] G. Zhao and N. Zhang, "Porous $\mathrm{MoO}_{3}$ films with ultra-short relaxation time used for supercapacitors," Materials Research Bulletin, vol. 48, no. 3, pp. 1328-1332, 2013.

[15] J. M. Liu, "Simple technique for measurements of pulsed Gaussian-beam spot sizes," Optics Letters, vol. 7, pp. 196-198, 1982.

[16] W. Wang, X. Mei, and G. Jiang, "Control of microstructure shape and morphology in femtosecond laser ablation of imprint rollers," International Journal of Advanced Manufacturing Technology, vol. 41, no. 5-6, pp. 504-512, 2009.

[17] A. Ben-Yakar, A. Harkin, J. Ashmore, R. L. Byer, and H. A. Stone, "Thermal and fluid processes of a thin melt zone during femtosecond laser ablation of glass: the formation of rims by single laser pulses," Journal of Physics D, vol. 40, no. 5, article 021, pp. 1447-1459, 2007.

[18] P. P. Pronko, S. K. Dutta, D. Du, and R. K. Singh, "Thermophysical effects in laser processing of materials with picosecond and femtosecond pulses," Journal of Applied Physics, vol. 78, no. 10, pp. 6233-6240, 1995.

[19] K. H. Leitz and B. Redlingshöfer, "Metal ablation with short and ultrashort laser pulses," Physics Procedia, vol. 12, pp. 230-238, 2011.

[20] K. C. Mills, S. U. Yuchu, L. I. Zushu, and R. F. Brooks, "Equations for the calculation of the thermo-physical properties of stainless steel," ISIJ International, vol. 44, no. 10, pp. 1661-1668, 2004.

[21] J. Stašic and B. Gakovic, "Superficial changes on the Inconel 600 superalloy by picosecond Nd:YAG laser operating at 1064, 532, and $266 \mathrm{~nm}$ : Comparative Study," Laser and Particle Beams, vol. 30, p. 249, 2012.

[22] E. G. Gamaly, A. V. Rode, B. Luther-Davies, and V. T. Tikhonchuk, "Ablation of solids by femtosecond lasers: ablation mechanism and ablation thresholds for metals and dielectrics," Physics of Plasmas, vol. 9, no. 3, p. 949, 2002.

[23] C. H. Fan, J. Sun, and J. P. Longtin, "Plasma absorption of femtosecond laser pulses in dielectrics," Journal of Heat Transfer, vol. 124, no. 2, pp. 275-283, 2002.

[24] R. Fedosejevs and R. Ottmann, "Absorption of femtosecond laser pulses in high-density plasma," Physical Review Letters, vol. 64, no. 11, pp. 1250-1253, 1990.

[25] J. König and T. Bauer, Fundamentals and Industrial Applications of Ultrashort Pulsed Lasers at Bosch, Photonics West, San Fransisco, Calif, USA, 2011.

[26] A. Miotello and R. Kelly, "Laser-induced phase explosion: new physical problems when a condensed phase approaches the thermodynamic critical temperature," Applied Physics A, vol. 69, no. 7, pp. S67-S73, 1999.

[27] L. M. Cabalin and J. J. Laserna, "Experimental determination of laser induced breakdown thresholds of metals under nanosecond Q-switched laser operation," Spectrochimica Acta B, vol. 53, no. 5, pp. 723-730, 1998.

[28] M. Momcilovica and J. Limpouch, "Surface modification of copper using high intensity, $10^{15} \mathrm{~W} / \mathrm{cm}^{2}$, femtosecond laser in vacuum," Applied Surface Science, vol. 258, p. 8908, 2012.

[29] S. Amoruso, R. Bruzzese, M. Vitiello, N. N. Nedialkov, and P. A. Atanasov, "Experimental and theoretical investigations of femtosecond laser ablation of aluminum in vacuum," Journal of Applied Physics, vol. 98, no. 4, Article ID 044907, 2005. 

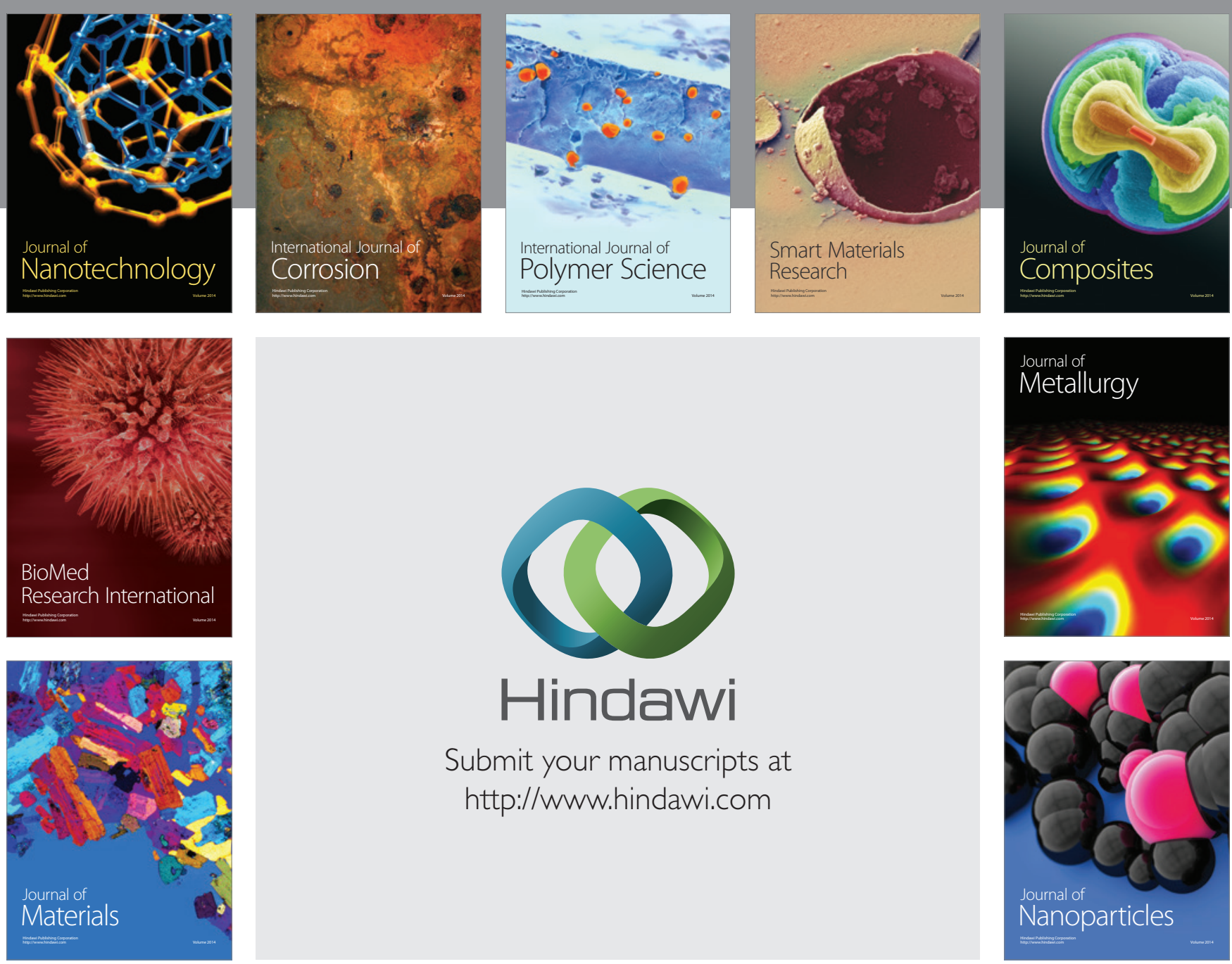

Submit your manuscripts at http://www.hindawi.com
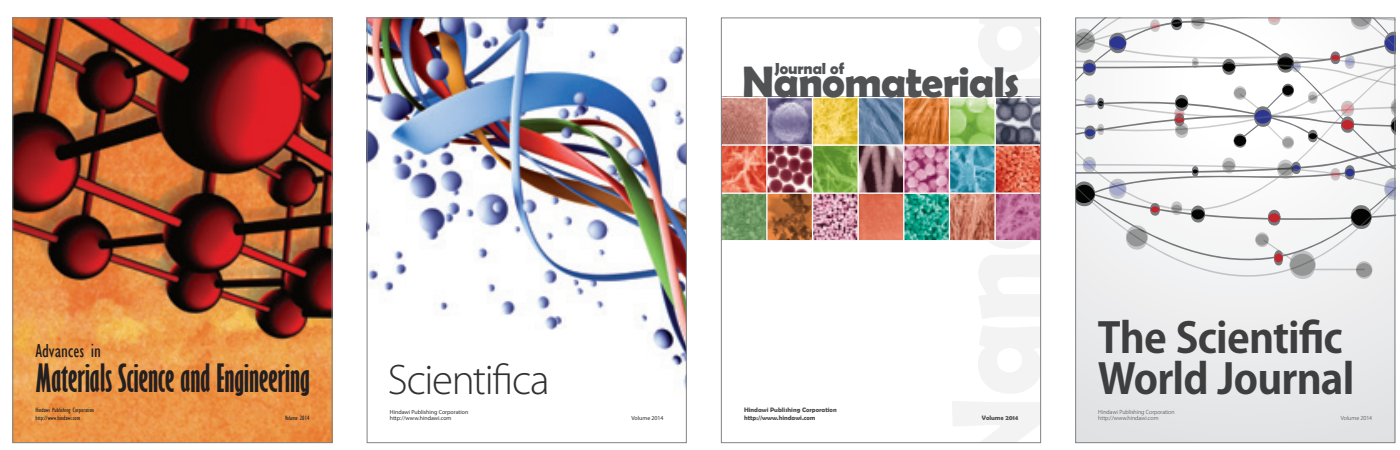

\section{The Scientific World Journal}
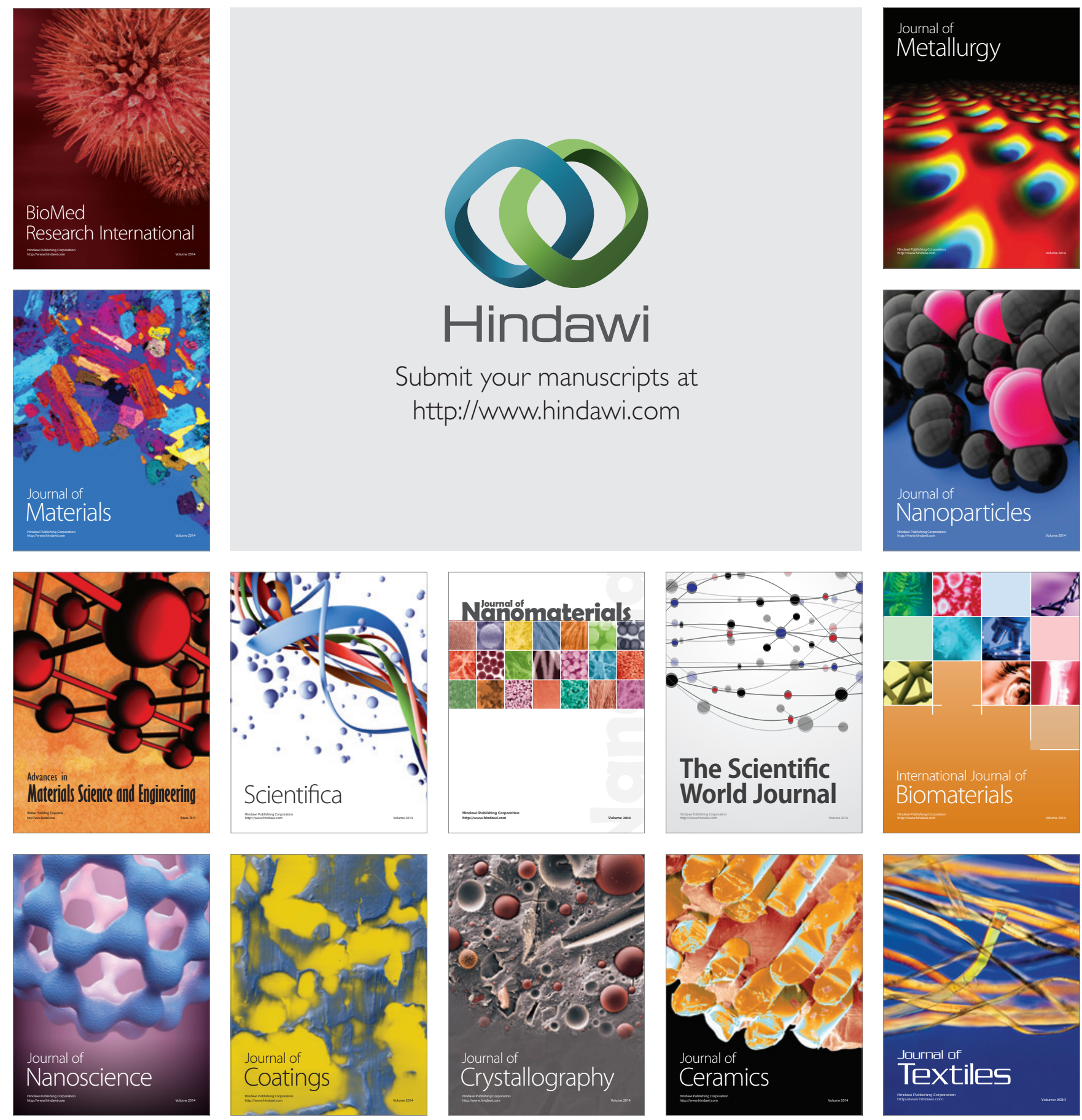